\title{
Breakup characteristics in plasma spraying of solution precursors and suspensions
}

\author{
Saman Shalbaf ${ }^{1}$, Mohammad Hashemi ${ }^{1}$, Mehdi Jadidi ${ }^{2}$, Ali Dolatabadi*1,3 \\ ${ }^{1}$ Department of Mechanical, Industrial and Aerospace Engineering, Concordia University, \\ Montreal, Canada \\ 2Department of Mechanical and Industrial Engineering, Ryerson University, Toronto, Canada \\ ${ }^{3}$ Department of Mechanical and Industrial Engineering, University of Toronto, Toronto, \\ Canada \\ *Corresponding author email: dolat@mie.utoronto.ca
}

\begin{abstract}
Solution precursor and suspension plasma spraying are emerging technologies to produce functional nanostructured coatings at moderate cost. In general, in these techniques, liquid is injected radially into a high-velocity high-temperature plasma flow. After liquid breakup and evaporation, solid particles remain in the field and impact the substrate. Preliminary studies have shown that liquid jet atomization is the main phenomenon that controls the coating quality. However, due to the complex thermophysical properties of plasma and its intricate flow physics, the breakup processes of liquid jets in plasma crossflows have not been investigated comprehensively yet. In general, the gaseous Reynolds number and the liquidto-gas density ratio in this process is around 50 and 10,000, respectively, which are far outside the limits normally observed in engines and wind tunnels. In the present work, detailed features of breakup phenomena of liquid jets in an argon plasma crossflow is provided. The finite volume scheme is used to solve the incompressible variable-density Navier-Stokes equations. In addition, volume of fluid (VOF) approach is utilized to track the gas-liquid interfaces. Overall, two different cases are simulated and different characteristics such as spray trajectory and droplet size distributions are investigated.
\end{abstract}

\section{Keywords}

Liquid jet in crossflow, Suspension, Solution, Plasma flow, Numerical simulation.

\section{Introduction}

To generate nanostructured coatings with superior properties, solution precursor and suspension plasma spraying techniques have been recently developed. By using these techniques, remarkable thermal barrier coatings, notable wear and corrosion resistance, as well as superhydrophobic surfaces have been generated [1]. Suspension is a combination of solid nano/submicron sized particles, a base liquid like water or ethanol, and a dispersant for stabilization. Solution is made by dissolving metal salts or precursors in a solvent [2]. In these techniques, first, suspension or solution precursor is injected into a high-velocity hightemperature plasma crossflow which breaks jet into the ligaments and droplets (see Figure 1). These chunks may undergo secondary breakup. Then, due to heat and mass transfer, the liquid phase is evaporated and the particulate phase is heated up. It is worth mentioning that in the solution precursor process, the particles are formed in flight during solvent evaporation. Finally, molten, semi-molten and solid particles, which have typically high amount of kinetic energy, impact the substrate and generate various types of coatings [3]. Preliminary experimental and numerical studies have shown that the coatings quality strongly depends on the liquid jet breakup [4]-[7]. Indeed, in addition to droplet size and velocity distributions, droplet position and trajectory play significant roles and must be controlled [4], [5]. 


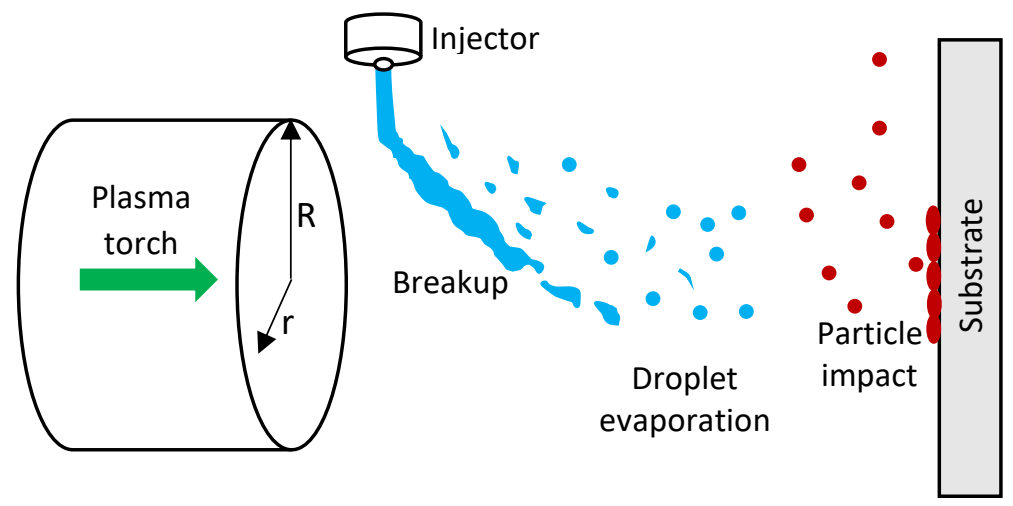

Figure 1. Phenomena involved in plasma spraying of solution precursors and suspensions.

In the field of liquid jet in crossflow, five nondimensional numbers are typically used to analyze the breakup characteristics. These five numbers are liquid Reynolds number $\left(R e_{l}\right)$, gaseous Reynolds number $\left(R e_{g}\right)$, gaseous Weber number $\left(W e_{g}\right)$, liquid-to-gas momentum flux ratio $(q)$, and liquid-to-gas density ratio $\left(r_{\rho}\right)$ [8], [9]. It should be noted that in the case of thermal plasma crossflow at atmospheric pressure, the plasma density is about one to two orders of magnitude (depending on the temperature and plasma composition) less than the air density. On the other hand, its viscosity is around one order of magnitude higher than the air viscosity [10]. As a result, $R e_{g}$ as well as $r_{\rho}$ are far from the observations in engines and wind tunnels. In addition, it should be pointed out that the profiles of plasma velocity and temperature at the torch exit are nonuniform; the temperature changes from $300 \mathrm{~K}$ to more than $12,000 \mathrm{~K}$ and the velocity varies from zero to about $1000-2000 \mathrm{~m} / \mathrm{s}$ [4]. Moreover, the plasma flow can oscillate and simultaneously swirl (depending on the operating condition and plasma composition) [11]-[13]. Furthermore, depending on the particle size, type, and concentration, as well as the dispersant type and concentration, the liquid can be non-Newtonian and the surface tension and viscosity can be time-dependent and dynamic [14].

The above discussion clearly shows that analyzing the behavior of suspensions and solution precursors in a thermal plasma crossflow is challenging since numerous parameters and phenomena are involved. Therefore, to better understand the effects of different parameters on the breakup mechanisms and to control the process and find the optimal operating condition, fundamental studies should be conducted. Performing experimental studies in thermal plasma is challenging due to high-frequency oscillations and high-intensity radiation of the plume. Consequently, numerical simulation, as a promising way to address the issues stated above, has attracted researchers' attention. So far, due to complexity of the problem and the computational cost, most numerical studies have been based on Eulerian-Lagrangian approach in which Taylor Analogy breakup (TAB) or Kelvin-Helmholtz Rayleigh-Taylor (KHRT) breakup models have been employed (for example, see [4], [5], [11]). In the TAB and KHRT models, several constants having significant influence on the breakup results exist [15]. It should be noted that estimating these constants for thermal plasma conditions has not been possible thus far due to lack of experimental data and absence of validated numerical and theoretical works. As a result, the values obtained from engine conditions have been simply used for these constants to simulate the liquid jet atomization in plasma crossflows (see [4], [5], [11]).

In the present study, numerical simulations are performed to show what breakup characteristics will (or will not) alter if we switch from an environment like a wind tunnel to a harsh situation such as argon plasma. For the breakup characteristics that are almost the 
same in both conditions, the experimental work can be done in a wind tunnel since it is faster and much less expensive. Conversely, for the characteristics that are very different, experiments in the plasma conditions need to be performed. In addition, since the numerical simulations in the current study are able to estimate different characteristics such as spray trajectory, droplet size and velocity distributions as well as droplet position and trajectory accurately, the results can be used to calibrate the constants in the KHRT and TAB models for the plasma conditions. In the following sections, the numerical approach and the results are explained.

\section{Methodology}

A mixture of immiscible liquid and gas substances is assumed to be incompressible at low Mach number. It should be noted that in a conventional atmospheric plasma spray the Mach number is typically less than 0.5 since the gas temperature is high. To perform the numerical simulations, a free open-source CFD code, called Basilisk [16], is used. In this code, the finite volume scheme is utilized to solve the Navier-Stokes equations:

$$
\nabla \cdot \boldsymbol{u}=0
$$

$$
\rho \frac{\partial \boldsymbol{u}}{\partial t}+\rho \boldsymbol{u} \cdot \nabla \boldsymbol{u}=-\nabla p+\boldsymbol{f}+\mu \nabla^{2} \boldsymbol{u}+\sigma \kappa \delta s \mathbf{n}
$$

where $\boldsymbol{u}$ is the flow velocity field, $p$ is the pressure, $\rho$ is the density, $\mu$ is the viscosity and $\boldsymbol{f}$ is the body force vector. The surface tension force is represented by the fourth term on the right side of Eq. (2), where a Dirac distribution function $\delta s$ indicates the interface. Here, $\sigma$ is the surface tension, $\boldsymbol{\kappa}$ is the local curvature and $\boldsymbol{n}$ is unit normal of interface. The volume of fluid (VOF) approach is applied to track the liquid-gas interface. The position of each substance is defined by a color function $C$ that takes the values of 1 and 0 for the liquid and gas phases, respectively. This function can be a fractional number at the interfaces. The interface position is determined by the solution of an advection equation for $C$ :

$$
\frac{\partial C}{\partial \mathrm{t}}+\mathbf{u} \cdot \nabla C=0
$$

In addition, the density and viscosity are correspondingly defined as,

$$
\begin{aligned}
& \rho=C \rho_{l}+(1-C) \rho_{g}, \\
& \mu=C \mu_{l}+(1-C) \mu_{g},
\end{aligned}
$$

where the variables with subscripts " $g$ " and " $l$ " show gas and liquid phases, respectively. The ones without a subscript are the local value based on the VOF approach [16].

In the Basilisk solver, the piecewise linear interface construction (PLIC) technique is utilized [17]. Moreover, the Bell-Colella-Glaz (BCG) method is implemented for the advection term [18]. The solver also uses a unique Octree adaptive mesh technique [19]. Furthermore, Basilisk has a tool named tag function which is developed to postprocess and assign an index to a ligament or droplet. This function is very useful to find the droplet size and velocity distributions. For further information about the Basilisk, the reader is referred to [16].

The computational domain in the current work is a $4.7 \times 4.7 \times 4.7 \mathrm{~cm}^{3}$. A large computational domain is chosen to capture the details of spray behavior in near-field and far-field. As shown in Figure 2, the $x$ and $y$ axes are in the directions of crossflow and liquid jet injection, 
respectively. Adaptive mesh refinement with 11 levels of refinement is also used to reduce the computational time and to model the turbulent flow accurately in both phases. The finest grid size in the present study reaches $\Delta x=22.9 \mu \mathrm{m}$. The center of liquid orifice is located at $(0.0$, $0.0,0.0)$. The uniform velocity profiles $\left(U_{g}\right.$ and $\left.U_{l}\right)$ are assumed the jet orifice and at $x=-$ $7.8 \mathrm{~mm}$ plane to simulate jet and crossflow inlets, respectively. The imposed boundary conditions are shown in Figure 2.

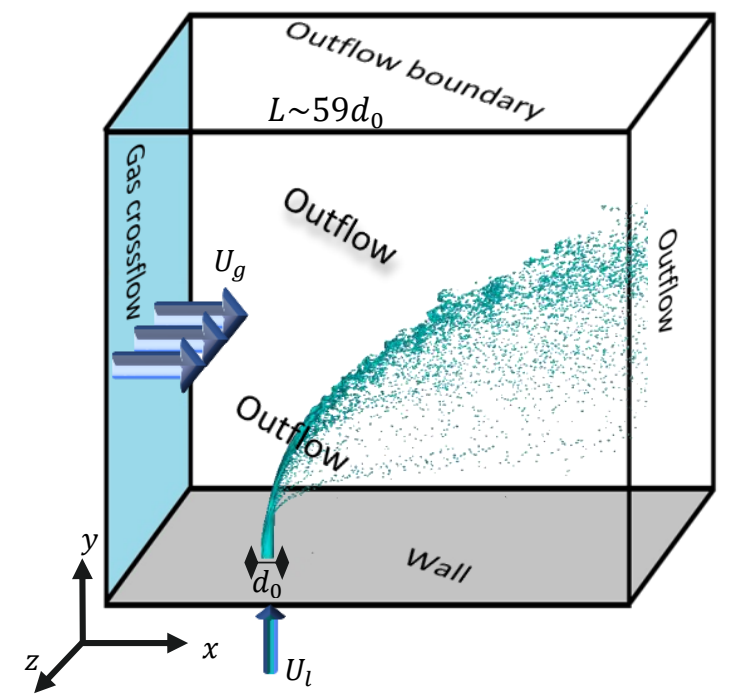

Figure 2. Schematic view of the cubic computational domain.

As mentioned above, in general, the velocity and temperature across the plasma crossflow are unsteady and nonuniform. However, for an argon plasma, the fluctuations can be negligible and the profiles are functions of space only [2], [13]. To perform the simulations, first, the profiles of velocity and temperature at the torch exit for an argon plasma flow were obtained from the work of Wan et al. [20]:

$$
\begin{aligned}
& u_{g}=u_{m}\left(1-\left(\frac{r}{R}\right)^{1.2}\right), u_{m}=1700 \mathrm{~m} / \mathrm{s} \\
& T_{g}=T_{a}+\left(T_{m}-T_{a}\right)\left(1-\left(\frac{r}{R}\right)^{6}\right), T_{a}=300 \mathrm{~K}, T_{m}=12,300 \mathrm{~K}
\end{aligned}
$$

where $r$ is the radial distance and $R$ is the exit radius of the plasma torch (see Figure 1) and is equal to $3.75 \mathrm{~mm}$ here. Then, the averages of gas density (it is a function of temperature), mass flux, and gas momentum at the exit of plasma torch were calculated. It was assumed that water interacts with the plasma crossflow. In addition, the liquid orifice diameter was assumed to be $0.25 \mathrm{~mm}$ which is consistent with the configuration of common suspension plasma spray systems [6], [20], [21].

Using the above assumptions, it was found that the average gaseous Reynolds number $\left(\overline{R e_{g}}\right)$, the average liquid-to-gas density ratio $\left(\overline{r_{\rho}}\right)$, and the average gaseous Weber number $\left(\overline{W e_{g}}\right)$ are 32, 11750, and 78, respectively. Similar results for $\overline{R e_{g}}$ and $\overline{r_{\rho}}$ were obtained in [22]. It should be pointed out that for a liquid jet in a crossflow under atmospheric condition in a wind tunnel the values of $R e_{g}$ and $r_{\rho}$ are significantly different (i.e. $R e_{g}$ is about two orders of magnitude higher and $r_{\rho}$ is one order of magnitude lower) [23]. In this regard, we increased the $R e_{g}$ to 3200 and decreased the $r_{\rho}$ to 1175 to have a new case representing the conditions in a wind tunnel. 
In suspension and solution precursor plasma spraying, the liquid mass flow rate depends on different parameters like particle concentration, however, a range of 20 to $40 \mathrm{~g} / \mathrm{min}$ is common [4], [5]. By using these values, it was found that $q$ and $R e_{l}$ are in the range of 2-11 and 15003800 , respectively. Due to the narrow range of variation, it was assumed that $q$ is equal to 10 and $R e_{l}$ is 2600 in the present study. Table 1 reveals a summary of the two cases investigated in the current work. As described, $R e_{g}$ and $r_{\rho}$ are different while other nondimensional numbers are fixed. In these simulations, $U_{g}=54.8 \mathrm{~m} / \mathrm{s}, \rho_{g}=1.18 \mathrm{~kg} / \mathrm{m}^{3}, \sigma=0.036 \mathrm{~N} / \mathrm{m}$, and $d_{0}=$ $8 \times 10^{-4} \mathrm{~m}$. It is worth mentioning that the values of density, viscosity, surface tension, velocity etc. were changed in such a way that the calculated nondimensional numbers were not altered but the numerical stability was guaranteed.

Table 1 - Thermo-fluid conditions for the two case studies

\begin{tabular}{|c|c|c|c|c|c|c|c|}
\hline Case & $R e_{g}$ & $R e_{l}$ & $q$ & $W e_{g}$ & $r_{\rho}$ & $\mu_{g}\left(N . s / m^{2}\right)$ & $U_{l}(m / s)$ \\
\hline 1. Plasma crossflow & 32 & 2600 & 10 & 78 & 11750 & $1.62 \times 10^{-3}$ & 1.6 \\
\hline 2. Air crossflow & 3200 & 2600 & 10 & 78 & 1175 & $1.62 \times 10^{-5}$ & 5.06 \\
\hline
\end{tabular}

\section{Results}

The spray boundaries from different views are shown in Figure 3. The left image shows the windward (solid line) and the leeward (dashed line) trajectories for both cases. As can be seen, the penetration height in case 2 is higher than that in case 1 . Moreover, compared to case 1 , the surface breakup starts slightly earlier in case 2 . The right image shows that the jet penetrations in the $z$ direction are the same. Figure 3 clearly reveals that to reasonably predict the spray trajectories of suspension and solution precursors in an argon plasma crossflow, the experiments can be conducted in a wind tunnel in such a way that $q, R e_{l}$, and $W e_{g}$ are kept constant. One of the existing empirical correlations can be also used to estimate the windward trajectory. However, one should be aware that the predictions of the available correlations are significantly different from each other. More information can be found in [24].
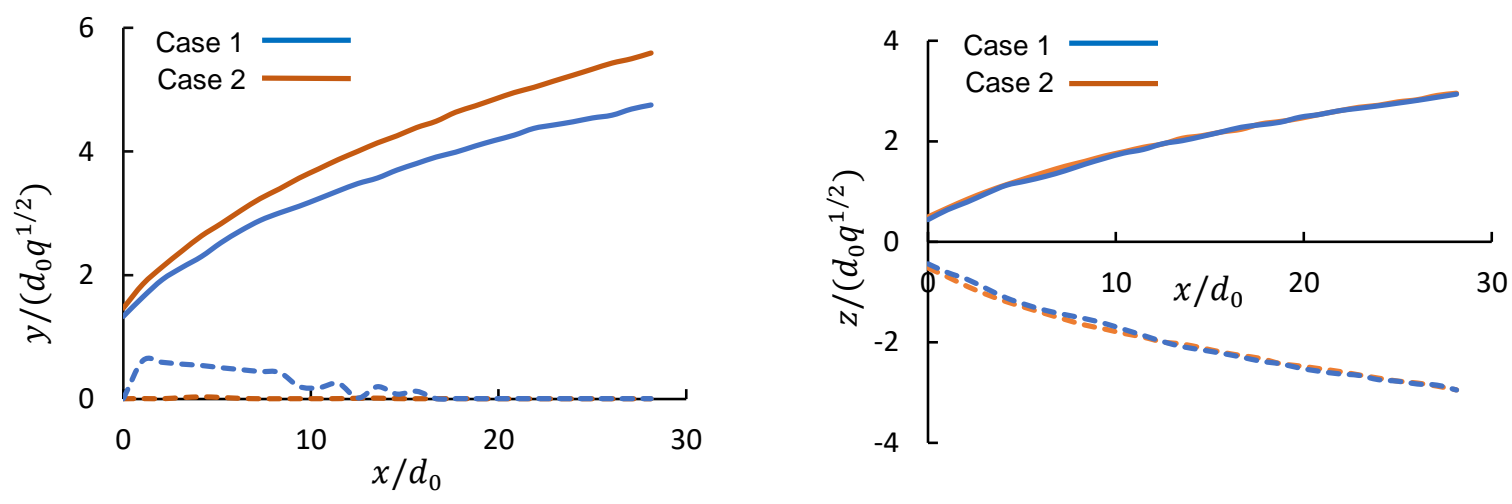

Figure 3. Comparison of spray plume boundaries

In Figure 4, the atomization features of liquid jets in crossflows for cases 1 and 2 are qualitatively compared in three orthogonal views. The snapshots of liquid jet breakup in $x-y$ frame (side view) $y-z$ frame (crossflow direction) and $x-z$ direction at the same physical time are demonstrated in this figure. It is observed that as the jet penetrates into the crossflow, it bends towards the crossflow stream. As can be seen, instability waves are formed on the column surface and result in the disintegration of small droplets and ligaments. Figure 4 also shows that the wave structure (e.g. their wavelengths and growth rates) in cases 1 and 2 is different, which causes the droplet size and velocity distribution to be dissimilar. 


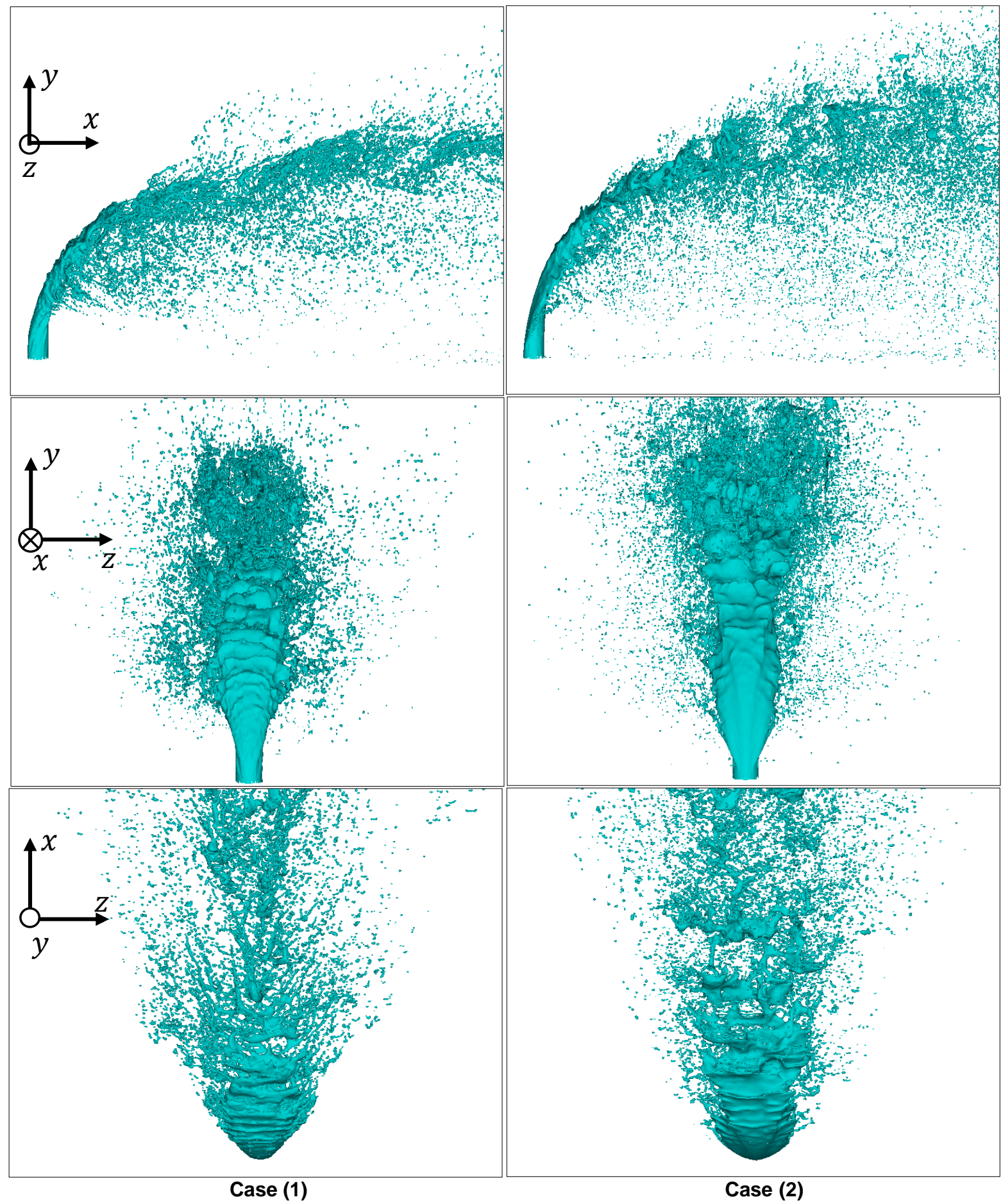

Figure 4. Snapshots of liquid jets in crossflow

To study the droplet statistics after jet atomization, the droplet size distribution in the forms of probability density functions (PDFs) are obtained using the tag function. In Figure 5, the droplet size distribution inside a $y-z$ plane with the thickness of $d_{0}$ at the distance of $20 d_{0}(16 \mathrm{~mm})$ from the orifice center (see the hashed area in the right image) is presented. As shown, the droplet size distribution in case 2 is much smaller than that in case 1 . The result proves that to obtain the droplet size distribution in suspension and solution precursor plasma sprays, we need to perform the experiments under plasma conditions. However, due to the harsh condition and the issues mentioned above, an alternative way is to develop more advanced numerical solvers and run more complicated simulations. 


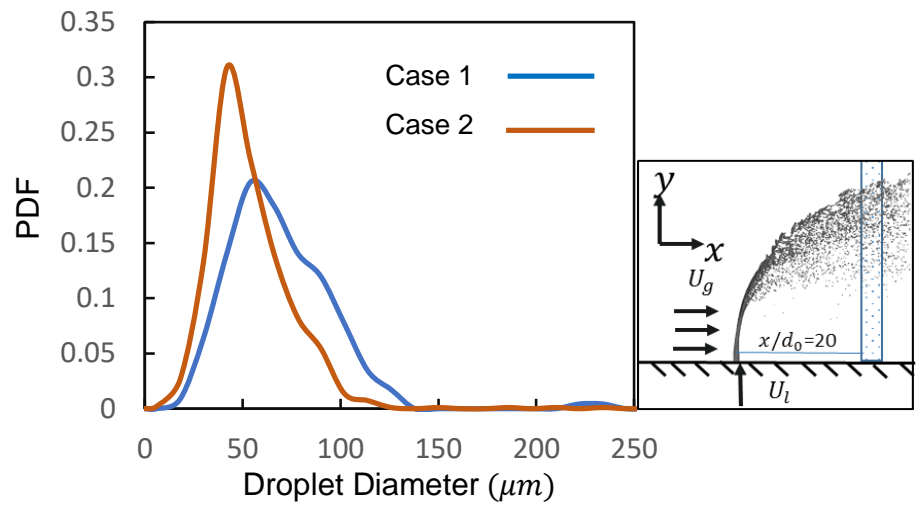

Figure 5. Comparison of droplet size distributions collected between planes $x / d_{0}=20$ and 21 .

In order to validate the numerical results, the droplet size distribution for case 2 is compared with the experimental data in the literature [25]. In this study, jet-A and water were injected into the air crossflow where $W e_{g}$ and $q$ were fixed at around 95 and 10, respectively. It was shown that at $x / d_{0}=30$, the SMD values for jet-A and water vary from 23.4-61.4 $\mu \mathrm{m}$ and 27$48.7 \mu \mathrm{m}$, respectively. As shown in Figure 5, in the present study, similar results have been obtained and very fine droplets as well as large ones (e.g. $100 \mu \mathrm{m})$ have been captured. It is worth mentioning that, compared to the work of Tambe [25], the droplet size in the current study is obtained at a closer distance $\left(x / d_{0}=20\right)$ and $W e_{g}$ is also lower $(=78)$. As a result, the presence of large droplets seems reasonable.

\section{Conclusions}

In this work, high-fidelity simulations were run to provide a detailed picture of atomization of suspension and solution precursor in thermal plasma crossflows. Two cases were considered. In case $1, R e_{g}, r_{\rho}, W e_{g}, R e_{l}$ and $q$ were obtained from the experiments in an argon plasma. In case 2, the $R e_{g}$ was increased and the liquid density was reduced to demonstrate the typical conditions in the wind tunnels. It was found that the spray trajectory in the plasma condition can be estimated reasonably by the findings from the wind tunnel tests. However, the results of droplet size distributions in cases 1 and 2 are significantly different. The current study provides a set of benchmark tests to tune the constants in the KHRT and TAB breakup models.

\section{Acknowledgments}

The authors acknowledge the Natural Sciences and Engineering Research Council of Canada (NSERC) for their financial support.

$\begin{array}{ll}\text { Nomenclature } \\ \mathrm{C} & \text { Color function } \\ \mathrm{d}_{\mathrm{o}} & \text { Injector orifice diameter [m] } \\ \boldsymbol{f} & \text { Body force vector } \\ \boldsymbol{n} & \text { Unit normal of interface } \\ q & \text { Momentum flux ratio } \\ r_{\rho} & \text { Liquid-to-gas density ratio } \\ R e & \text { Reynolds number } \\ t & \text { Time [s] } \\ \boldsymbol{u} & \left.\text { Velocity vector [m. } \mathrm{s}^{-1}\right] \\ U & \left.\text { Imposed velocity [m. } \mathrm{s}^{-1}\right] \\ W e & \text { Weber number }\end{array}$


$x \quad$ Coordinate in the crossflow direction

$y \quad$ Coordinate in the liquid injection direction

$z \quad$ Coordinate orthogonal to $x$ and $y$

$\kappa \quad$ Local curvature

$\mu \quad$ Dynamic viscosity [N.s.m $\left.{ }^{-2}\right]$

$\rho \quad$ Density $\left[\mathrm{kg} \cdot \mathrm{m}^{-3}\right]$

$\sigma \quad$ Surface tension $\left[\mathrm{N} . \mathrm{m}^{-1}\right]$

Subscripts

g Gas

I Liquid

\section{References}

[1] Fauchais, P., Vardelle, M., Goutier, S., Vardelle, A., 2015, Plasma Chemistry and Plasma Processing, 35(3), pp. 511-525.

[2] Jadidi, M., Vardelle, A., Dolatabadi, A., Moreau, C., 2018, "Heat Transfer in Suspension Plasma Spraying," in Handbook of Thermal Science and Engineering, Springer International Publishing, pp. 2923-2966.

[3] Pawlowski, L., 2009, Surface and Coatings Technology, 203(19), pp. 2807-2829.

[4] Jabbari, F., Jadidi, M., Wuthrich, R., Dolatabadi, A., 2014, Journal of Thermal Spray Technology, 23(1-2), pp. 3-13.

[5] Jadidi, M., Mousavi, M., Moghtadernejad, S., Dolatabadi, A., 2015, Journal of Thermal Spray Technology, 24, pp. 11-23.

[6] Fazilleau, J., Delbos, C., Rat, V., Coudert, J.F., Fauchais, P., Pateyron, B., 2006, Plasma Chemistry and Plasma Processing, 26, pp. 371-391.

[7] Fauchais, P., Rat, V., Coudert, J.F., Etchart-Salas, R., Montavon, G., 2008, Surface and Coatings Technology, 202(18), pp. 4309-4317.

[8] Herrmann, M., 2010, Journal of Engineering for Gas Turbines and Power, 132(6), p. 061506.

[9] Herrmann, M., 2011, Proceedings of the Combustion Institute, 33(2), pp. 2079-2088.

[10] Fauchais, P.L., Heberlein, J.V.R., Boulos, M.I., 2014, "Thermal Spray Fundamentals."

[11] Dalir, E., Dolatabadi, A., Mostaghimi, J., Journal of Thermal Spray Technology, 28(6), pp. 1105-1125.

[12] Coudert, J.F., Planch, M.P., Fauchais, P., 1995, Plasma Chemistry and Plasma Processing, 15(1), pp. 47-70.

[13] Trelles, J.P., Pfender, E., Heberlein, J., 2006, Plasma Chemistry and Plasma Processing, 26, pp. 557-575.

[14] Jadidi, M., Moghtadernejad, S., Dolatabadi, A., 2015, Coatings, 5(4), pp. 576-645.

[15] ANSYS Inc., 2013, "Ansys Fluent Theory Guide."

[16] Popinet, S., 2015, Journal of Computational Physics, 302, pp. 336-358.

[17] Scardovelli, R., and Zaleski, S., 1999, Annual Review of Fluid Mechanics, 31, pp. 567603.

[18] Bell, J.B., Colella, P., Glaz, H.M., 1989, Journal of Computational Physics, 85(2), pp. 257283.

[19] Popinet, S., 2003, Journal of Computational Physics, 190(2), pp. 572-600.

[20] Wan, Y.P., Sampath, S., Prasad, V., Williamson, R., Fincke, J.R., 2003, Journal of Materials Processing Technology, 137(1-3), pp. 110-116.

[21] Siegert, R., 2005, Bochum Univ. Diss., "A Novel Process for the Liquid Feedstock Plasma Spray of Ceramic Coatings with Nanostructural Features."

[22] Sun, X., and Heberlein, J., 2005, Journal of Thermal Spray Technology, 14, pp. 39-45.

[23] Li, X., and Soteriou, M.C., 2016, Physics of Fluids, 28, p. 082101.

[24] No, S.Y., 2015, International Journal of Spray and Combustion Dynamics, 7(4), pp. 283314.

[25] S. B. Tambe, "Liquid Jets in Subsonic Crossflow," University of Cincinnati, 2004. 